\title{
Manifestações da apraxia de fala na doença de Alzheimer
}

\section{Manifestations of apraxia of speech in Alzheimer's disease}

\author{
Maysa Luchesi Cera ${ }^{1}$, Karin Zazo Ortiz², Paulo Henrique Ferreira Bertolucci ${ }^{3}$, Thaís Soares Cianciarullo Minett ${ }^{2}$
}

\section{RESUMO}

\begin{abstract}
Objetivo: Identificar as manifestações práxicas de pacientes com doença de Alzheimer em diferentes estágios da doença e verificar as similaridades entre as suas ocorrências. Métodos: Foram avaliados noventa pacientes com doença de Alzheimer, 30 em cada fase da doença (leve, moderada e grave), por meio dos instrumentos: Escala de Avaliação Clínica da Demência (CDR), Mini-Exame do Estado Mental (MEEM), Avaliação das Atividades Instrumentais de Vida Diária (Índice Lawton) e Protocolo de Avaliação da Apraxia Verbal e Não-verbal. Foram avaliados 66 pacientes do gênero feminino e 24 do masculino, a média da idade foi 80,2 27,2 e da escolaridade foi 4,2 $\pm 3,5$ anos. Resultados: Na fase leve, as proporções de ensaio, repetição e adição foram semelhantes, assim como omissão, substituição e autocorreção. Na fase moderada foram semelhantes: ensaio e repetição, substituição, omissão e adição, e autocorreção. Na fase grave, todas as manifestações se assemelharam, exceto a adição. O erro do tipo adição diferenciou os pacientes em relação às fases da doença. Conclusão: Os pacientes dos três estágios da doença apresentam diferentes padrões de manifestações práxicas verbais.
\end{abstract}

Descritores: Apraxias/diagnóstico; Doença de Alzheimer/complicações; Demência; Transtornos da articulação; Testes neuropsicológicos

\section{INTRODUÇÃO}

Segundo o Instituto Brasileiro de Geografia e Estatística (IBGE), o envelhecimento da população está se acentuando: em 2000, a população brasileira com mais de 65 anos era de apenas 5\%; estima-se que em 2050 será de $18 \%$. Além disso, em 2050, estima-se que a expectativa de vida, ao nascimento, será de $81,3 \operatorname{anos}^{(1)}$.

O aumento da expectativa de vida da população ocasionou maior prevalência e incidência de doenças neurodegenerativas e, em especial, da doença de Alzheimer (DA), que é o tipo de demência mais frequente ${ }^{(2)}$.

Um estudo brasileiro mostrou uma prevalência de demência de 1,3\% dos 65 aos 69 anos, que eleva-se para 36,9\% nas idades superiores à 84 anos. Entre os diagnósticos de demência mais frequentes, a DA representou $54,1 \%$ e DA associada à demência vascular, $14,4 \%$ (2).

Trabalho realizado no Departamento de Fonoaudiologia, Universidade Federal de São Paulo - UNIFESP - São Paulo (SP), Brasil.

(1) Programa de Pós-graduação (Mestrado) em Distúrbios da Comunicação Humana, Universidade Federal de São Paulo - UNIFESP - São Paulo (SP), Brasil.

(2) Departamento de Fonoaudiologia, Universidade Federal de São Paulo UNIFESP - São Paulo (SP), Brasil.

(3) Departamento de Neurologia e Neurocirurgia, Universidade Federal de São Paulo - UNIFESP - São Paulo (SP), Brasil.

Endereço para correspondência: Maysa Luchesi Cera. R. Botucatu, 802, Vila Clementino, São Paulo (SP), Brasil, CEP: 04023-900. E-mail: mamaysa@hotmail.com

Recebido em: 26/5/2010; Aceito em: 20/10/2010
A DA é uma doença degenerativa progressiva do sistema nervoso central que causa deterioração da memória e de pelo menos uma das seguintes funções cognitivas: orientação, linguagem, praxia, atenção, percepção visual e funções executivas $^{(3)}$. Seu diagnóstico se baseia no quadro clínico e na exclusão de outras causas de demência por meio de exames laboratoriais e só pode ser confirmado por exames neuropatológicos. No entanto, os critérios estruturados para o diagnóstico clínico oferecem concordância com o diagnóstico neuropatológico de até $90 \%{ }^{(3)}$. Assim, a avaliação clínica tem extrema importância para o diagnóstico precoce e, desta forma, a avaliação dos aspectos cognitivos é de grande valia.

As apraxias são frequentemente descritas como manifestação das demências, inclusive da $\mathrm{DA}^{(4)}$. Alguns relatos de casos foram descritos em pacientes com apraxia verbal ${ }^{(5,6)}$ e os estudos com maiores casuísticas se referem aos outros tipos de apraxia, como as apraxias de membros e ideatória ${ }^{(7)}$.

A apraxia verbal foi descrita como um distúrbio que ocasiona prejuízo da capacidade para programar o posicionamento da musculatura da fala e de sequencializar os movimentos durante a produção voluntária de fonemas ${ }^{(8)}$.

Este estudo é parte de uma dissertação de mestrado que mostrou que os pacientes com DA, independente do estágio da doença, apresentaram apraxia de fala, que progrediu com a evolução da demência ${ }^{(9)}$.

Uma melhor caracterização das manifestações da apraxia verbal em indivíduos com DA poderá auxiliar na avaliação precoce dos transtornos de comunicação e no seguimento destes pacientes. 
Assim, o objetivo deste estudo foi analisar as manifestações práxicas do grupo de pacientes com DA, nos diferentes estágios da doença e verificar as similaridades entre as suas ocorrências.

\section{MÉTODOS}

Este trabalho foi submetido à avaliação do Comitê de Ética em Pesquisa da Universidade Federal de São Paulo e recebeu aprovação (protocolo número 0390/08). Antes da participação na pesquisa foi obtida a autorização dos indivíduos e responsáveis por eles, por meio do Termo de Consentimento Livre e Esclarecido, que foi elaborado em respeito às normas da Resolução número 196, de 10 de outubro de 1996, do Conselho Nacional de Saúde.

Trata-se de um estudo transversal que se baseou em uma amostra de 90 idosos com DA: 30 na fase leve, 30 na moderada e 30 na fase grave da doença, matriculados em ambulatório do setor de Neurologia do Comportamento. Dos 90 indivíduos analisados, 66 eram mulheres. A idade variou de 64 a 97 anos, com média de $80,2 \pm 7,2$ anos e a escolaridade de zero a 12 anos, com média de 4,2 $\pm 3,5$ anos.

Os critérios de inclusão dos pacientes foram: idade maior ou igual a 60 anos; diagnóstico de DA provável, segundo os critérios clínicos de NINCDS-ADRDA ${ }^{(3)}$; estarem acompanhados de um cuidador que pudesse servir como informante. Além disso, os participantes com DA leve e moderada deveriam estar em tratamento para DA com inibidores da acetilcolinesterase em dose terapêutica (Donepezil $\geq 5 \mathrm{mg}$, Rivastigmina $\geq 9 \mathrm{mg}$ ou Galantamina $\geq 8 \mathrm{mg})^{(10)}$, conforme as diretrizes governamentais de distribuição das drogas para o tratamento da DA na rede pública (Ministério da Saúde - Secretaria de Assistência à Saúde - Portaria no 843 , de 31 de outubro de 2002) $)^{(11)}$. Como a droga memantina não foi contemplada na portaria $\mathrm{n}^{\circ} 843$, não foi critério de inclusão o recebimento desta medicação pelos pacientes com DA grave.

Os critérios de exclusão foram: história de alcoolismo, ou uso de drogas ilícitas; uso de drogas psicoativas (uso por pelo menos uma vez no último mês, exceto os neurolépticos atípicos); doenças graves neurológicas ou psiquiátricas prévias (ex.: epilepsia, carcinoma, esquizofrenia); alterações visuais ou auditivas que pudessem comprometer o desempenho nas tarefas realizadas; ausência de emissão oral ou impossibilidade de responder à avaliação, uma vez que para analisar a fala era necessário que o paciente respondesse aos estímulos.

Todos os pacientes foram acompanhados por uma equipe multidisciplinar composta por neurologistas, psicólogos e fonoaudiólogos. Quando as escalas relacionadas a esta pesquisa não haviam sido aplicadas na avaliação médica ou psicológica no dia da avaliação fonoaudiológica, estas foram aplicadas pela fonoaudióloga responsável pelo estudo, assim como o protocolo fonoaudiológico. Foram aplicadas as escalas descritas a seguir.

- Escala clínica de demência - Clinical Dementia Rating $(\mathrm{CDR})^{(12,13)}$. Esta escala avalia a cognição e o comportamento, além da influência das perdas cognitivas na capacidade do indivíduo realizar adequadamente atividades de vida diária. Neste instrumento o desempenho atual é comparado ao passado, de tal modo que os próprios indivíduos servem para a referência de comparação. Apresenta seis categorias (memória, orientação, julgamento ou solução de problemas, relações comunitárias, atividades no lar ou de lazer e cuidados pessoais). A fase da doença é classificada de acordo com o escore total do teste, que considera a memória como categoria principal e as demais categorias como secundárias. Se a pontuação total do CDR for igual a um, significa demência leve; dois, demência moderada e três, demência grave.

- Mini-Exame do Estado Mental (MEEM) ${ }^{(14)}$. Teste que serve como rastreio para alteração cognitiva. É composto por questões sobre orientação temporal e espacial, memória imediata, cálculo, evocação de palavras, nomeação, repetição, comando oral e escrito, frase e cópia de desenho, com escore mínimo de zero e máximo de 30 pontos, em que os escores maiores representam melhores desempenhos. Todos os pacientes apresentaram desempenho no MEEM abaixo dos padrões estabelecidos para a população brasileira $^{(15)}$.

- Índice de Lawton ${ }^{(16)}$. Avalia as atividades instrumentais da vida diária (AIVD), tais como usar o telefone, fazer compras, executar atividades domésticas, usar transporte, tomar medicamentos, controlar finanças, preparar refeições e andar fora de casa. Cada um dos itens da escala apresenta pontuação de zero a dois (zero para independência, um para dependência parcial, dois para dependência total), e a soma dos valores dos itens classifica o indivíduo em independente ou dependente. São considerados independentes os sujeitos com pontuação zero ou um e dependentes para as demais pontuações.

\section{Avaliação fonoaudiológica}

Para a caracterização dos movimentos práxicos de fala dos sujeitos foram utilizadas as tarefas verbais do protocolo de avaliação da apraxia verbal e não-verbal ${ }^{(17)}$. A avaliação da apraxia verbal deste protocolo inclui tarefas de repetição de palavras, repetição de frases, emissão de automatismos, fala espontânea e leitura em voz alta. Para eliciar a fala espontânea foi utilizada a Prancha "Roubo dos Biscoitos" do Teste de Boston para o Diagnóstico da Afasia ${ }^{(18)}$ e foram gravados dois minutos da emissão oral do paciente. No Brasil não há um protocolo de avaliação da apraxia com tarefas foneticamente balanceadas para a língua portuguesa, pois é fundamental que o protocolo tenha as variáveis que influenciam o desempenho de pacientes com apraxia de fala em tarefas emissivas, como: os erros são mais frequentes em palavras compostas por fonemas menos frequentes na língua, palavras mais extensas ou com grupos consonantais, palavras com fonemas fricativos e que contêm fonemas com distantes pontos articulatórios. Assim, as tarefas deste protocolo contemplam tais variáveis e, por este motivo, não são balanceadas foneticamente.

Durante todas as tarefas da avaliação da apraxia verbal, a emissão oral dos sujeitos foi gravada e transcrita concomitantemente, para maior precisão na coleta dos dados. A transcrição da fala foi realizada durante a avaliação para a adequada classificação do tipo de manifestação da apraxia de fala, uma vez que o ensaio articulatório pode ocorrer sem a 
fonação, quando o sujeito busca o movimento fonoarticulatório do fonema desejado sem vocalizar.

Para a análise do desempenho verbal foram registrados o número e os tipos de manifestações práxicas cometidas durante todas as provas da avaliação da apraxia verbal do protocolo.

Inicialmente, a emissão oral de cada paciente foi transcrita e computou-se a quantidade e o tipo de cada manifestação. Foram consideradas as manifestações típicas da emissão oral de indivíduos com apraxia de fala: substituição, repetição, omissão, adição, autocorreção, ensaio articulatório:

- Substituição: troca de um fonema por outro;

- Omissão: não produção de um fonema ou sílaba na palavra;

- Adição: acréscimo de um fonema ou sílaba na palavra;

- Autocorreção: correção espontânea de erros práxicos. As autocorreções de idéias e conteúdos, que são realizadas com frequência pelos pacientes com $\mathrm{DA}^{(19)}$, não foram consideradas manifestações de fala, pois se referem à alteração de linguagem;

- Ensaio articulatório: busca do ponto articulatório de um fonema ou da sequência de fonemas, na procura do movimento adequado antes de sua produção;

- Repetição: mais de uma emissão de um som, parte da palavra, palavra ou parte do enunciado.

Quando o indivíduo cometeu mais de uma manifestação em uma sílaba ou palavra, todas foram computadas. As falas foram transcritas e analisadas subjetivamente pela principal autora do estudo. Quando houve dúvida, a segunda autora realizou o julgamento.

Pelo fato de os pacientes deste estudo também apresentarem erros de conteúdo no discurso, apenas as repetições de seguimentos das palavras (repetição de som ou de parte da palavra) foram consideradas para o computo das manifestações práxicas. Pela frequente ocorrência de repetições de palavra e de parte de enunciado relacionadas à alteração discursiva em pacientes com $\mathrm{DA}^{(19)}$, estes erros não foram considerados na análise de fala. Além disso, as hesitações ocorridas na fala espontânea foram desconsideradas, uma vez que esta manifestação pode estar presente na fala de indivíduos normais e de pacientes com alteração de linguagem ou de fala.

\section{Método estatístico}

A fim de identificarmos a similaridade entre a quantidade dos tipos de manifestações foi realizada a análise de agrupamento (cluster analysis). As variáveis foram padronizadas em
Z escores. O coeficiente de distância Euclidiana foi calculado para mensurar as dissimilaridades entre as variáveis. O método de agrupamento utilizado foi o de Ward. A sequência derivada desta análise foi visualmente representada por um dendograma. Este diagrama representa graficamente o quão perto ou distante cada item está dos outros, baseado em uma hierarquia de similaridades. Isto é, itens muito similares estão pertos uns dos outros, enquanto itens dissimilares estão distantes.

Os dados foram analisados no software estatístico SPSS (Statistical Package for the Social Science), versão 13.0.

\section{RESULTADOS}

A média do escore do MEEM para o estágio leve foi de $20,0 \pm 3,4$, para o estágio moderado foi de $14,4 \pm 1,6$ e para o grave 9,0 2 2,9. A média do Índice Lawton para os pacientes com DA leve foi de $7,0 \pm 4,2$; moderada $14,3 \pm 2,0$ e grave $15,4 \pm 1,0$.

A Tabela 1 apresenta as médias do número de manifestações de praxia verbal do grupo DA e dos pacientes dos três estágios da doença: leve, moderado e grave. A média de manifestações práxicas verbais dos pacientes com DA grave foi maior, em relação à média dos pacientes com DA moderada, que foi maior que do grupo leve.

Tabela 1. Análise descritiva do número de manifestações de emissão oral da apraxia verbal, de acordo com o estágio da doença

\begin{tabular}{lcccccc}
\hline PV & $\mathrm{n}$ & Média & $\mathrm{DP}$ & Mínimo & Máximo & Mediana \\
\hline DA & 90 & 11,4 & 8,8 & 0,0 & 39,0 & 9,0 \\
Leve & 30 & 6,0 & 4,2 & 0,0 & 16,0 & 6,0 \\
Moderada & 30 & 13,0 & 6,9 & 0,0 & 26,0 & 12,0 \\
Grave & 30 & 15,3 & 11,1 & 1,0 & 39,0 & 11,5
\end{tabular}

Legenda: $\mathrm{DP}=$ desvio-padrão; $\mathrm{DA}=$ doença de Alzheimer; $\mathrm{PV}=$ número de manifestações na tarefa de praxia verbal do protocolo de avaliação da apraxia verbal e não-verbal

A Tabela 2 apresenta a análise descritiva das manifestações práxicas verbais cometidas pelos pacientes nas três fases da DA. Em geral, a ordem de frequência das manifestações observada em todos os grupos foi: substituição, omissão, ensaio articulatório, repetição, autocorreção e adição, respectivamente.

As Figuras 1, 2, 3 e 4 apresentam os resultados das análises de agrupamento realizadas pelo método de Ward dos tipos de manifestações práxicas apresentadas pelos pacientes com DA.

Os agrupamentos dos tipos de manifestações formados pelos pacientes com DA foram: Grupo 1 - ensaio articulatório

Tabela 2. Análise descritiva dos tipos de manifestações práxicas verbais apresentadas pelos pacientes com DA

\begin{tabular}{lcccccccc}
\hline Tipos de erros & \multicolumn{2}{c}{ DA } & \multicolumn{2}{c}{ Leve } & \multicolumn{2}{c}{ Moderado } & \multicolumn{2}{c}{ Grave } \\
\cline { 2 - 8 } & Média & DP & Média & DP & Média & DP & Média & DP \\
\hline Substituição & 3,4 & 3,3 & 1,6 & 1,9 & 4,5 & 3,7 & 4,1 & 3,4 \\
Omissão & 3,5 & 3,3 & 2,1 & 2,0 & 4,4 & 3,9 & 4,0 & 3,4 \\
Ensaio & 2,6 & 3,0 & 1,3 & 1,3 & 2,4 & 2,0 & 4,1 & 4,1 \\
Autocorreção & 0,5 & 0,6 & 0,3 & 0,5 & 0,6 & 0,7 & 0,5 & 0,6 \\
Repetição & 1,1 & 2,1 & 0,6 & 1,0 & 0,7 & 1,1 & 2,1 & 3,2 \\
Adição & 0,4 & 0,7 & 0,2 & 0,5 & 0,3 & 0,7 & 0,6 & 0,9 \\
\hline
\end{tabular}

Legenda: $\mathrm{DA}$ = doença de Alzheimer; $\mathrm{DP}$ = desvio-padrão 
e repetição; Grupo 2 - substituição e omissão; Grupo 3 - autocorreção; Grupo 4 - adição (Figura 1).

Os agrupamentos dos tipos de manifestações formados pelos pacientes do estágio leve foram: Grupo 1 - ensaio articulatório, repetição e adição; Grupo 2 - omissão, autocorreção e substituição (Figura 2).

Os agrupamentos dos tipos de manifestações formados pelos pacientes com DA moderada foram: Grupo 1 - ensaio articulatório e repetição; Grupo 2 - substituição, omissão e adição; Grupo 3 - autocorreção (Figura 3).

Os agrupamentos dos tipos de manifestações formados pelos pacientes com DA grave foram: Grupo 1 - ensaio articulatório, repetição, substituição, omissão; autocorreção; Grupo 2 - adição (Figura 4).

\section{DISCUSSÃO}

O resultado mais relevante deste estudo foi que os pacientes

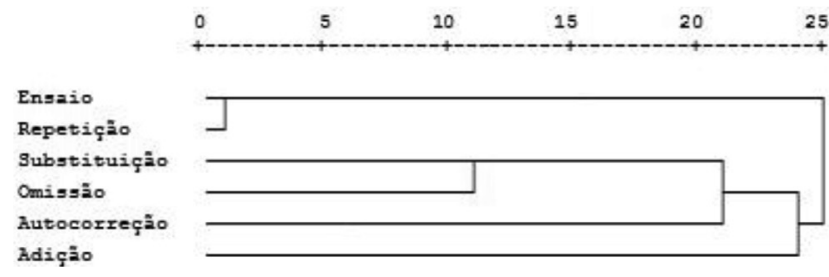

Figura 1. Dendograma obtido na análise de agrupamento pelo método de Ward dos tipos de manifestações do grupo DA

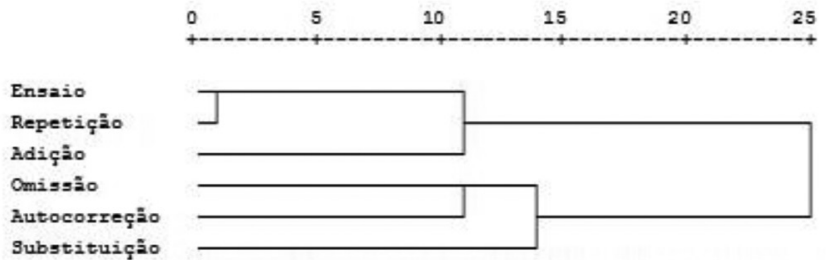

Figura 2. Dendograma obtido na análise de agrupamento pelo método de Ward dos tipos de manifestações apresentadas pelos pacientes com DA leve

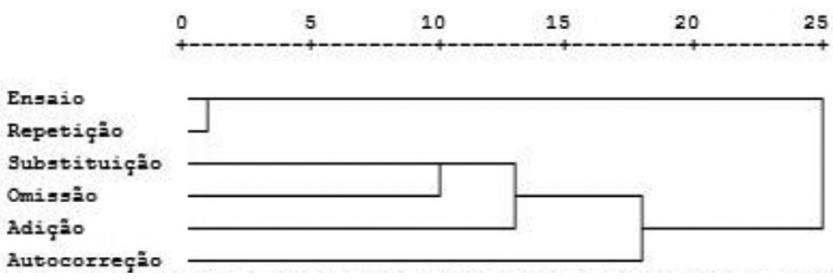

Figura 3. Dendograma obtido na análise de agrupamento pelo método de Ward dos tipos de manifestações dos pacientes com DA moderada

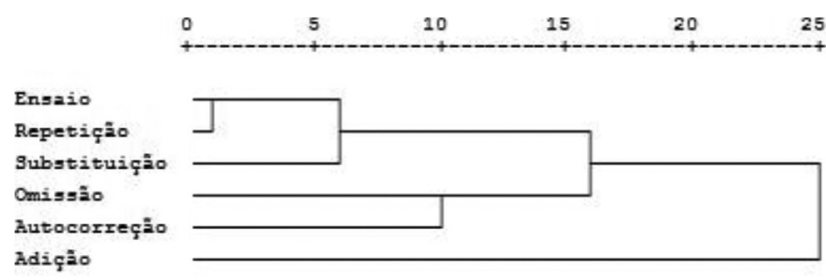

Figura 4. Dendograma obtido na análise de agrupamento pelo método de Ward dos tipos de manifestações dos pacientes com DA grave com DA apresentaram diferentes padrões de manifestações práxicas verbais de acordo com a fase da doença.

Observamos que os pacientes do estágio leve apresentaram menor frequência de manifestações práxicas, ou seja, desempenho menos prejudicado em relação aos pacientes dos estágios mais avançados da doença (Tabela 1). Este resultado pode ser justificado pela evolução da doença, pois mais áreas cerebrais são afetadas e há uma piora do quadro cognitivo geral e, consequentemente, das manifestações práxicas verbais.

Quanto aos tipos de manifestações (Tabela 2), parece que os tipos substituição, omissão, ensaio articulatório e repetição são os mais característicos de pacientes com DA. Este achado está de acordo com estudos sobre a apraxia verbal na DA e em outras doenças ${ }^{(20,21)}$.

O desempenho articulatório de dez pacientes com apraxia de fala foi analisado e comparado com dez pacientes com disartria e dez sujeitos normais ${ }^{(20)}$. Os tipos de manifestações encontradas nos pacientes com apraxia de fala foram: substituição (32\%), repetição (18\%), intrusão (18\%), outros erros não classificados (12\%), distorção (10\%), adição (9\%) e omissão $(<1 \%)$. Observa-se que, assim como em nosso estudo, a substituição foi um dos erros que apresentou maior frequência e a adição uma das menores.

A substituição é uma manifestação característica da apraxia de fala e foi descrita anteriormente em um estudo de caso de uma paciente com diagnóstico anátomo-patológico de DA que apresentou manifestações iniciais evidentes de apraxia de fala ${ }^{(6)}$.

Já o erro do tipo omissão, um dos mais frequentes em nosso estudo, apresentou ocorrência diferente em outro estudo $^{(20)}$. Esta diferença em relação ao nosso estudo pode estar relacionada à terminologia dos tipos de manifestações e à amostra estudada, uma vez que incluímos pacientes com DA falantes do Português e neste estudo foram avaliados pacientes pós AVC falantes do inglês. E, por outro lado, em relação à diferença de terminologia para a classificação do tipo de erro omissão, um estudo encontrou predomínio do erro do tipo distorção, seguido de omissão ${ }^{(22)}$. A segunda maior frequência encontrada neste estudo também foi do erro omissão, porém estes autores incluíram o erro do tipo distorção, que não foi incluído em nosso estudo. Neste estudo, a dessonorização foi classificada em erro do tipo distorção, enquanto, em nosso estudo, consideramos este erro como substituição, uma vez que o fonema sonoro é substituído pelo surdo.

Há autores que classificam a distorção como uma produção imprecisa de um fonema que é reconhecível ${ }^{(20)}$. Não consideramos este erro na análise da amostra de fala dos pacientes, pois, apesar de termos considerado as manifestações típicas da apraxia de fala, não há consenso da classificação deste tipo de erro, conforme apresentado acima. Além disso, a distorção conforme a classificação descrita acima $^{(22)}$ não foi observada em nosso estudo.

Em estudos brasileiros ${ }^{(21-23)}$, em que foi usada a mesma terminologia, as manifestações substituição, omissão e ensaio articulatório apresentaram as maiores frequências na fala dos pacientes que sofreram AVC, seguidas de repetição e autocorreção. O mesmo foi observado em nosso estudo, o que parece confirmar a influência nas habilidades práxicas verbais 
em relação aos aspectos fonético-fonológicos de cada língua, que possui corpus de fonemas, combinatórias e frequências diferentes de uso $^{(23)}$.

As diferenças podem ser justificadas também pelos materiais e estímulos usados para a avaliação destes pacientes, em que a frequência de fonemas e as ocorrências de estímulos com diferentes complexidades são distintas tanto na língua quanto no protocolo de avaliação utilizado, conforme demonstrado em estudos prévios ${ }^{(21-23)}$. Além disso, a estrutura da Língua Portuguesa é distinta da inglesa, na qual são realizados a maioria dos estudos de indivíduos com apraxia de fala. Estudos mostraram interferência da complexidade da sílaba inicial e da frequência da sílaba na programação motora de pacientes com apraxia de fala $^{(24,25)}$. A diversidade na estrutura e na frequência das sílabas de acordo com a língua pode contribuir para a diferenciação da frequência dos tipos de manifestações.

A análise de agrupamento mostrou que as manifestações do tipo ensaio articulatório e repetição apresentaram ocorrência similar em todos os estágios da DA (Figuras 2, 3 e 4).

A ocorrência similar de omissão, substituição e autocorreção na fase leve, pode ser justificada pela menor gravidade da doença, provavelmente porque nesta fase, os pacientes ainda são capazes de perceber e corrigir seus erros de substituição e omissão. No entanto, na fase moderada da DA, com a piora do quadro cognitivo geral, este resultado não se manteve.

Em relação às outras manifestações, observamos que a ocorrência do erro do tipo adição mostrou-se diferente nas diversas fases da doença. É interessante notar que, apenas na fase grave da DA, todos os tipos de manifestações se assemelharam, exceto a adição. O que pode justificar este resultado é que, com a progressão da doença, a ocorrência de todas as manifestações aumenta e tende a aparecer em todos os pacientes. O erro do tipo adição, apesar de sua menor frequência na fala de pacientes com DA, pode ser mais suscetível às variações da doença. Isso porque, na fase leve, a adição foi similar ao ensaio articulatório e à repetição e, na fase moderada, à substituição e omissão. A adição foi a manifestações menos frequente na fala dos pacientes com DA. Encontramos achados distintos em um estudo internacional sobre a apraxia de fala, que verificou que este tipo de manifestação apresentou maior frequência em relação à omissão e autocorreção ${ }^{(26)}$ e foi semelhante ao estudo brasileiro descrito acima, que usou uma metodologia similar e avaliou uma população com a mesma língua ${ }^{(21)}$. Assim, a discrepância pode estar relacionada não apenas à diferença etiológica para a alteração de fala, mas também à progressão da doença.

Por outro lado, a menor frequência do tipo de erro adição está de acordo com estudo sobre análise fonológica dos erros de indivíduos afásicos ${ }^{(27)}$, que mostraram erros de substituição e omissão mais frequentes que adição. Desta forma, observamos que o padrão de erros difere, nos diferentes estudos. Assim, o erro do tipo adição pode ser mais susceptível a variações segundo a população estudada e a metodologia empregada.

As manifestações da DA dependerão dos locais de acometimento da lesão. Sugerimos que o estágio e as manifestações clínicas da doença, bem como a ocorrência e a gravidade da apraxia de fala dependerão da lesão cerebral. Ou seja, quanto maior o comprometimento cerebral, mais manifestações poderão ocorrer, bem como poderá haver um maior prejuízo de alterações já existentes, uma vez que mais áreas cerebrais envolvidas na função da fala estarão comprometidas. Além disso, o aumento do número de manifestações conforme a gravidade da doença apoia outra hipótese, do envolvimento de um maior comprometimento da memória de trabalho no decorrer da doença.

Estudos sobre a apraxia de fala também discutem a relação entre este distúrbio e a memória de trabalho ${ }^{(28)}$, que está alterada na DA por disfunção da alça fonoarticulatória. A alteração da reverberação subvocal e da manutenção subvocal das informações na alça fonoarticulatória pode prejudicar, inclusive, a realização de autocorreções dos erros. Esta dificuldade de autocorreção dos erros foi observada nos pacientes avaliados neste estudo.

As autocorreções durante a fala parecem envolver o processamento da retroalimentação da informação. Um estudo sobre o monitoramento visuomotor sugeriu que a apraxia de fala é resultante de alteração do processo de pro-alimentação do controle motor ${ }^{(29)}$, conceituado no modelo DIVA de processamento de fala ${ }^{(30)}$. Quando há perturbações, esse sistema necessita do controle de retroalimentação. Apesar dos pacientes avaliados neste estudo terem apresentado autocorreções, eles provavelmente apresentam alteração, inclusive, no uso do sistema de retroalimentação, uma vez que a ocorrência de autocorreções nem sempre esteve presente na ocorrência dos erros de substituição, omissão e adição.

Os pacientes no estágio leve apresentaram menor frequência de manifestações práxicas, ou seja, desempenho menos prejudicado em relação aos pacientes dos estágios mais avançados da doença, por isso, levantamos a hipótese de que eles apresentam maior evidência de realização de revisão anterior à produção do erro. Isto indica que eles parecem ser mais capazes de compensar suas dificuldades em relação aos pacientes dos estágios moderado e grave.

As manifestações de fala durante a evolução da DA de um caso foi descrita ${ }^{(6)}$. Os autores constataram erros de substituição e omissão no quinto ano da doença e, com o tempo, o paciente passou a apresentar mais erros articulatórios. No nono ano da doença, apresentou importante redução comunicativa. Observamos, em nosso estudo, que, em termos gerais, os erros de substituição e omissão apresentaram maior similaridade. No entanto, não encontramos estudos sobre os tipos de manifestações da apraxia de fala na DA, além de descrições de estudos de casos únicos, para compararmos com os resultados obtidos no presente estudo.

A descrição das manifestações da apraxia de fala realizadas neste estudo contribui para a acurácia da avaliação fonoaudiológica dos distúrbios da comunicação, bem como auxilia o planejamento terapêutico de pacientes com apraxia de fala. Assim, terá implicações para a prática clínica e para futuras pesquisas.

Uma limitação do estudo é que a amostra não incluiu pacientes com DA que apresentavam redução ou supressão de fala, pois estes foram critérios de exclusão, uma vez que não teríamos como analisar as manifestações da fala. Assim, talvez o padrão de manifestações dos pacientes com DA grave seja diferente do encontrado em nosso estudo. A redução ou supressão de fala impediriam a realização da análise de fala e não permitiriam a comparação do desempenho práxico 
verbal entre os três estágios da doença. Além disso, o número de manifestações poderia ser superestimado em pacientes com emissão mais fluente, uma vez que a probabilidade de ocorrência de erros aumentaria.

Outro fator limitante foi a não realização da análise da tarefa de leitura, uma vez que os pacientes apresentaram erros linguísticos que interferiram na análise práxica verbal. A coocorrência de distúrbios linguísticos e práxicos impediria a precisão e fidelidade da análise específica dos erros de origem práxica na leitura.

\section{CONCLUSÃO}

O erro do tipo adição determinou mudanças no padrão de manifestações encontradas nas diferentes fases da DA. As diferenças práxicas verbais observadas entre os três estágios da doença podem auxiliar na avaliação clínica e no diagnóstico da DA, bem como no acompanhamento longitudinal da doença e, consequentemente, nas condutas do tratamento medicamentoso e de reabilitação.

\section{AGRADECIMENTOS}

Agradecemos ao Conselho Nacional de Desenvolvimento Científico e Tecnológico (CNPq) e à Fundação de Amparo à Pesquisa do Estado de São Paulo (FAPESP), pelos apoios concedidos para realização desta pesquisa, sob processos números 132979/2008-0 e 2008/52670-1, respectivamente.

\begin{abstract}
Purpose: To identify praxic speech manifestations in patients diagnosed with different stages of Alzheimer's disease, and to verify similarities among their occurrences. Methods: Participants were 90 patients with Alzheimer's, 30 in each stage of the disease (mild, moderate and severe), who were assessed using the following instruments: Clinical Dementia Rating (CDR), Mini-Mental State Examination (MMSE), Lawton Instrumental Activities of Daily Living, and the Assessment Protocol of Verbal and Orofacial Apraxia. Sixty six female subjects and 24 male subjects were assessed; mean age was $80.2 \pm 7.2$ years, and mean level of education was $4.2 \pm 3.5$ years. Results: In the mild stage, the proportions of trial-and-error, repetition and addition were similar, as well as omission, substitution and self-correction. In the moderate stage, the following items were similar: trial-and-error and repetition, substitution, omission and addition, and self-correction. In the severe stage, all manifestations were similar, except addition. The error type addition differentiated the patients regarding the stages of the disease. Conclusion: Patients in the three stages of Alzheimer's disease showed distinct patterns of verbal praxic manifestations.
\end{abstract}

Keywords: Apraxia/diagnosis; Alzheimer disease/complications; Dementia; Articulation disorders; Neuropsychological tests

\title{
REFERÊNCIAS
}

1. Instituto Brasileiro de Geografia e Estatística. Projeção da população do Brasil: 1980-2050 [Internet] [citado 2008 Mar 12]. Disponível em: $<$ http://www.ibge.gov.br/>. Acesso em: 12/03/2008.

2. Herrera Júnior E, Caramelli P, Nitrini R. Estudo epidemiológico populacional de demência na cidade de Catanduva - Estado de São Paulo - Brasil. Rev Psiquiatr Clín (São Paulo). 1998;25(2):70-3.

3. McKhann G, Drachman D, Folstein M, Katzman R, Price D, Stadlan EM. Clinical diagnosis of Alzheimer's disease: report of the NINCDSADRDA Work Group under the auspices of Department of Health and Human Services Task Force on Alzheimer's Disease. Neurology. 1984;34(7):939-44.

4. Derouesné C, Lagha-Pierucci S, Thibault S, Baudouin-Madec V, Lacomblez L. Apraxic disturbances in patients with mild to moderate Alzheimer's disease. Neuropsychologia. 2000;38(13):1760-9.

5. Croot K, Hodges JR, Xuereb J, Patterson K. Phonological and articulatory impairment in Alzheimer's disease: a case series. Brain Lang. 2000;75(2):277-309.

6. Gerstner E, Lazar RM, Keller C, Honig LS, Lazar GS, Marshall RS. A case of progressive apraxia of speech in pathologically verified Alzheimer disease. Cogn Behav Neurol. 2007;20(1):15-20.

7. Crutch SJ, Rossor MN, Warrington EK. The quantitative assessment of apraxic deficits in Alzheimer's disease. Cortex. 2007;43(7):976-86.

8. Darley FL. Nomenclature of expressive speech-language disorders [abstract]. [Presented at Academy of Aphasia Meeting; 1969 Sept 30; Boston, Massachusetts].

9. Cera ML. Apraxia de fala e apraxia não-verbal na doença de Alzheimer [tese]. São Paulo: Universidade Federal de São Paulo; 2010.

10. Birks J. Cholinesterase inhibitors for Alzheimer's disease. Cochrane Database Syst Rev. 2006;(1):CD005593.

11. Brasil. Ministério da Saúde. Secretaria de Assistência à Saúde. Portaria n. 843, de 31 de outubro de 2002. Aprova o protocolo clínico e diretrizes terapêuticas - Demência por Doença de Alzheimer [Internet]. Diário Oficial da República Federativa do Brasil, Brasília (DF); 2002 Out 31 [citado 2009 Set 8]. Disponível em: http://sna.saude.gov.br/legisla/ legisla/informes/SAS_P843_02informes.doc

12. Morris JC. The Clinical Dementia Rating (CDR): current version and scoring rules. Neurology. 1993;43(11):2412-4.

13. Montaño MB, Ramos LR. Validade da versão em português da Clinical Dementia Rating. Rev Saúde Pública. 2005;39(6):912-7.

14. Folstein MF, Folstein SE, McHugh PR. "Mini-mental state". A practical method for grading the cognitive state of patients for the clinician. J Psychiatr Res. 1975;12(3):189-98.

15. Brucki SM, Nitrini R, Caramelli P, Bertolucci PH, Okamoto IH. Sugestões para o uso do mini-exame do estado mental no Brasil. Arq Neuro-Psiquiatr. 2003;61(3B):777-81.

16. Lawton MP, Brody EM. Assessment of older people: self-maintaining and instrumental activities of daily living. Gerontologist. 1969;9(3):17986.

17. Martins FC, Ortiz KZ. Proposta de protocolo para avaliação da apraxia de fala. Fono Atual. 2004;30:53-61.

18. Goodglas H, Kaplan EF. The assessment of aphasia and related disorders. 2nd ed. Philadelphia (PA): Lea \& Febiger; 1983. 
19. de Lira JO, Ortiz KZ, Campanha AC, Bertolucci PH, Minett TS. Microlinguistic aspects of the oral narrative in patients with Alzheimer's disease. Int Psychogeriatr. 2011;23(3):404-12.

20. Johns DF, Darley FL. Phonemic variability in apraxia of speech. J Speech Hear Res. 1970;13(3):556-83.

21. Cera ML, Minett TS, Ortiz KZ. Analysis of error type and frequency in apraxia of speech among portuguese speakers. Dement Neuropsychol. 2010;4(2):98-103.

22. Odell K, McNeil MR, Rosenbek JC, Hunter L. Perceptual characteristics of consonant production by apraxic speakers. J Speech Hear Disord. 1990;55(2):345-59.

23. Cera ML, Ortiz KZ. Análise fonológica dos erros da apraxia adquirida de fala. Pró Fono. 2009;21(2):143-8.

24. Riecker A, Brendel B, Ziegler W, Erb M, Ackermann H. The influence of syllable onset complexity and syllable frequency on speech motor control. Brain Lang. 2008;107(2):102-13.
25. Aichert I, Ziegler W. Syllable frequency and syllable structure in apraxia of speech. Brain Lang. 2004;88(1):148-59.

26. Deal JL, Darley FL. The influence of linguistic and situational variables on phonemic accuracy in apraxia of speech. J Speech Hear Res. 1972;15(3):639-53.

27. Romani C, Olson A, Semenza C, Granà A. Patterns of phonological errors as a function of a phonological versus an articulatory locus of impairment. Cortex. 2002;38(4):541-67.

28. Martins FC, Ortiz KZ. The relationship between working memory and apraxia of speech. Arq Neuropsiquiatr. 2009;67(3B):843-8.

29. Robin DA, Jacks A, Hageman C, Clark HM, Woodworth G. Visuomotor tracking abilities of speakers with apraxia of speech or conduction aphasia. Brain Lang. 2008;106(2):98-106.

30. Guenther FH, Ghosh SS, Tourville JA. Neural modeling and imaging of the cortical interactions underlying syllable production. Brain Lang. 2006;96(3):280-301. 